\title{
Thickness independence of true phase transition temperatures in barium strontium titanate films
}

\author{
A. Lookman, R. M. Bowman, and J. M. Gregg a) \\ Department of Pure \& Applied Physics, Queen's University Belfast, Belfast BT7 1NN, United Kingdom \\ J. Kut, S. Rios, M. Dawber, A. Ruediger, and J. F. Scott \\ Symetrix Centre for Ferroics, Department of Earth Science, University of Cambridge, Cambridge CB2 3EQ, \\ United Kingdom
}

(Received 25 November 2003; accepted 10 April 2004)

\begin{abstract}
The functional properties of two types of barium strontium titanate (BST) thin film capacitor structures were studied: one set of structures was made using pulsed-laser deposition (PLD) and the other using chemical solution deposition. While initial observations on PLD films looking at the behavior of $T_{m}$ (the temperature at which the maximum dielectric constant was observed) and $T_{c}^{*}$ (from Curie-Weiss analysis) suggested that the paraelectric-ferroelectric phase transition was progressively depressed in temperature as BST film thickness was reduced, further work suggested that this was not the case. Rather, it appears that the temperatures at which phase transitions occur in the thin films are independent of film thickness. Further, the fact that in many cases three transitions are observable, suggests that the sequence of symmetry transitions that occur in the thin films are the same as in bulk single crystals. This new observation could have implications for the validity of the theoretically produced thin film phase diagrams derived by Pertsev et al. [Phys. Rev. Lett. 80, 1988 (1998)] and extended by Ban and Alpay [J. Appl. Phys. 91, 9288 (2002)]. In addition, the fact that $T_{m}$ measured for virgin films does not correlate well with the inherent phase transition behavior, suggests that the use of $T_{m}$ alone to infer information about the thermodynamics of thin film capacitor behavior, may not be sufficient. (C) 2004 American Institute of Physics.
\end{abstract}

[DOI: 10.1063/1.1759084]

\section{INTRODUCTION}

It has been known for many years that the measured functional properties of ferroelectric thin films are strongly dependent on thickness. ${ }^{1}$ For example, as film thickness is reduced: strong increases in coercive field occur ${ }^{2-4}$ (though in some cases appear to saturate in extremely thin films $\left.{ }^{4,5}\right)$ : catastrophic reductions in dielectric constant ${ }^{6}$ are observed; "Curie anomalies" in dielectric constant normally seen in bulk and single crystal are progressively suppressed, and increase in diffuseness; ${ }^{7}$ the temperature at which the dielectric constant is at a maximum $\left(T_{m}\right)$ can change dramatically, ${ }^{7,8}$ and ferroelectricity itself can be lost. ${ }^{9,10}$ Unfortunately, although considerable debate has taken place, the origins of such "size effects" are still not fully understood, despite the technological imperative of integrating thin film ferroelectrics into data storage devices. ${ }^{11,12}$

In this paper, the authors present a series of functional property observations on barium strontium titanate (BST) thin film capacitor structures made using both pulsed-laser deposition (PLD) and chemical solution deposition (CSD) techniques. At first sight, measurements seemed to be largely consistent with the size effects described above. However, further investigations suggested the following.

(i) $T_{m}$ (the temperature at which the observed dielectric constant is maximum) measured on virgin films is not necessarily simply related to phase transition behavior, and can-

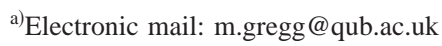

not therefore be reliably used as a phase transition monitor in thin film systems. This means that interpretations of thin film behavior such as those given recently by Parker et ll $^{7}$ need to be considered with care.

(ii) Three phase transitions are generally displayed by the thin films, which would be the case in bulk as symmetry changes from cubic to tetragonal to orthorhombic to rhombohedral. Such similarities to bulk behavior may have implications for the validity of recent theoretical work by Pertsev et $a l .{ }^{13}$ and extended by Ban and Alpay ${ }^{14}$ in the treatment of real thin film systems.

\section{EXPERIMENTAL METHOD}

\section{A. Pulsed-laser deposited films}

$\mathrm{Ba}_{0.5} \mathrm{Sr}_{0.5} \mathrm{TiO}_{3}$-based thin film capacitors (Au/BST/ $\mathrm{La}_{0.5} \mathrm{Sr}_{0.5} \mathrm{CoO}_{3}[\mathrm{LSCO}]$ ), with varying thickness of BST layer, were produced by PLD on commercially available $\mathrm{MgO}\{100\}$ substrates. A KrF excimer laser (Lambda Physik COMPex 205i) with $\lambda=248 \mathrm{~nm}$, energy density of $\sim 2$ $\mathrm{J} \mathrm{cm}^{-2}$ on the target surface, repetition rate of $10 \mathrm{~Hz}$, and a target-substrate distance of $75 \mathrm{~mm}$ was used. The pressure in the growth chamber was maintained at $0.15 \mathrm{mbar}_{2}$ and the deposition temperature at $650{ }^{\circ} \mathrm{C}$ for both the lower electrode and the dielectric layer. A postdeposition anneal for $30 \mathrm{~min}$ in $0.15 \mathrm{mbar}_{2}$ and $20 \mathrm{~min}$ at $1 \mathrm{bar}_{2}$ at $550{ }^{\circ} \mathrm{C}$ was used. Thermal evaporation of $\mathrm{Au}$ top electrodes through a hard mask enabled functional measurements by contacting two gold pads and measuring two capacitors in series. The elec- 


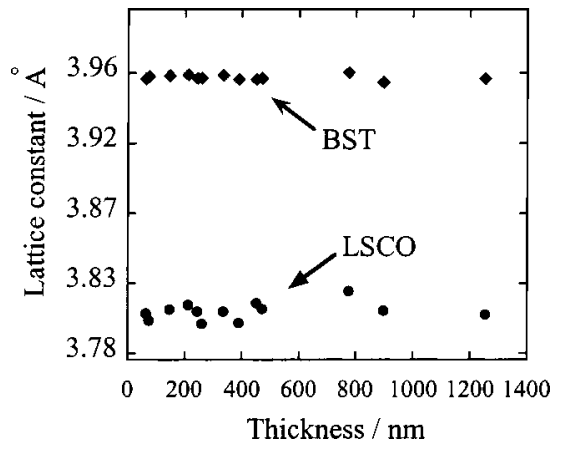

FIG. 1. Out-of-plane lattice parameters for the BST and LSCO as a function of BST film thickness. No effects of strain coupling were evident, suggesting interfacial relaxation of misfit strain through semicoherence.

trode area was $\sim 1.77 \times 10^{-6} \mathrm{~m}^{2}$. Low-field capacitance and the dielectric loss tangents were measured using a HP 4284A Precision LCR meter and HP 4263B LCR meter. Polarization measurements were taken using a Radiant Technologies Precision Workstation. For varying temperature, capacitors were mounted in an Oxford Instruments cryostat with temperature regulated using a calibrated Pt thermometer and Lakeshore 330 temperature controller. All data acquisition and instrument control was achieved using HPVEE software. A Tecnai F20 field-emission transmission electron microscope (FEGTEM) was used for microstructural characterization including film-thickness information, with cross-sectional specimens made in a focused ion beam microscope (FEI FIB200TEM). X-ray diffraction (XRD) was performed on a Brüker-AXS D8 instrument at room temperature.

\section{B. Chemical solution deposited films}

$175 \mathrm{~nm}$ thick $\mathrm{Ba}_{0.7} \mathrm{Sr}_{0.3} \mathrm{TiO}_{3}$-based thin film capacitor structures were produced in Juelich by Dr. Susana HoffmanEifert via a propionate-based chemical solution deposition (CSD) with 48 ex situ gold sputtered $\left(0.1 \mathrm{~mm}^{2}\right)$ top electrodes and a continuous Pt [111] bottom electrode on oxidized Si [111] substrates. Zero-bias permittivity studies were made on these structures with a Hewlett-Packard 4192A impedance analyzer. Temperature-variable XRD measurements were carried out using $\mathrm{Cu} K_{\alpha 1}$ radiation and a onedimensional position sensitive detector.

\section{RESULTS AND DISCUSSION}

\section{A. Pulsed-laser deposited films}

\section{Experimental observations}

The out-of-plane lattice parameter of the BST films was monitored using $\theta-2 \theta \mathrm{XRD}$, and was found to be independent of film thickness (Fig. 1). This was in contrast to previous work using $\mathrm{SrRuO}_{3}$ lower electrodes, ${ }^{8,15}$ and was extremely useful, as it allowed changes in functional behavior with film thickness to be studied without the complications and uncertainties associated with accounting for straincoupling effects. It appears that any homogeneous strain associated with the BST-LSCO interface has been relieved over a short distance and that the nature of the interface is

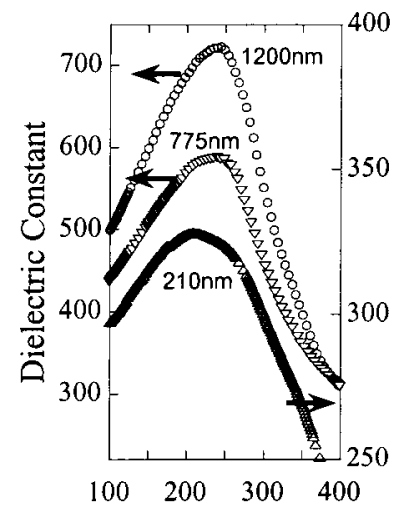

(a) Temperature / $\mathrm{K}$

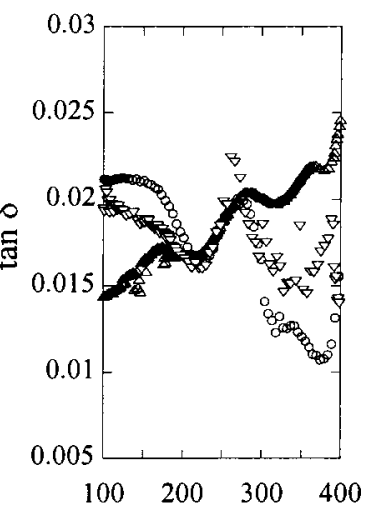

(b) Temperature / $\mathrm{K}$
FIG. 2. The dielectric constant (a) and loss tangent (b) for three of the thin film capacitors illustrating the decrease in dielectric constant, and also the migration of $T_{m}$, as BST thickness was reduced.

semicoherent rather than fully coherent. Indeed, in crosssectional TEM, "stitching" from misfit dislocations was frequently observed.

The low-field dielectric constant was recorded as a function of the temperature and frequency at different BST thicknesses, and several features were immediately apparent: the dielectric constant decreased with decreasing thickness (Fig. $2)$; the temperature at which the maximum dielectric constant was observed $\left(T_{m}\right)$ migrated to lower temperatures (Figs. 2 and 3) and the diffuseness of the peak in dielectric constant with temperature increased (Fig. 4) on decreasing thickness, as parametrized by fits used by Parker et al. ${ }^{7}$ (note the correction to the Taylor expansion in Eq. $(1)^{16}$ as per Smolenski ${ }^{17}$ ):

$$
\begin{aligned}
\frac{1}{\varepsilon} & =\frac{1}{\varepsilon_{\max }}+\frac{\left(T-T_{\max }\right)^{2}}{2 \varepsilon_{\max } \delta^{2}}, \\
\varepsilon & =\beta_{0}+\beta_{1} T+\beta_{2} T^{2} .
\end{aligned}
$$

All of these features are commonly observed size-related effects.

Curie-Weiss plots $[1 / \varepsilon(T)$ against $T]$ seen in Fig. 5 were produced from the temperature dependence of the dielectric constant, and the changes in the apparent Curie Temperature

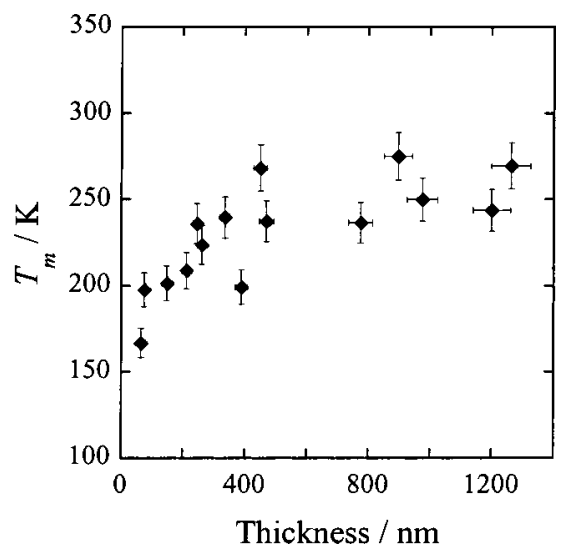

FIG. 3. The temperature at which the dielectric constant reached a maximum $\left(T_{m}\right)$ as a function of BST film thickness. 


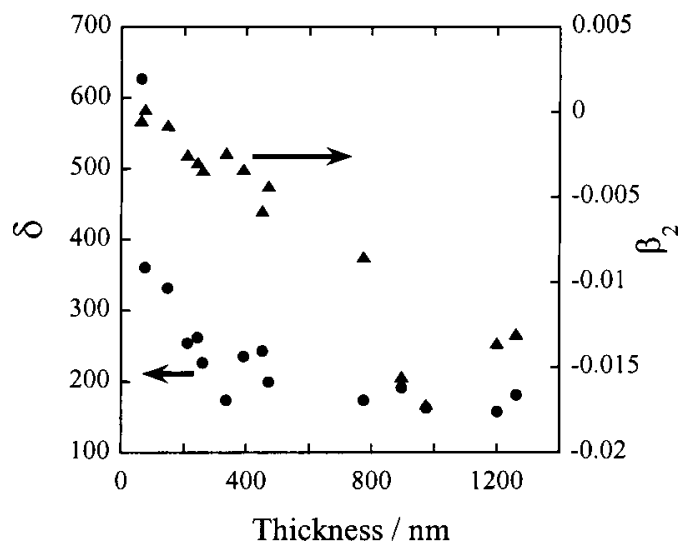

FIG. 4. When the variation in dielectric constant with temperature was fitted to various equations used in previous literature to quantify "diffuseness" of peak, it was found that the diffuseness increased systematically on reduction of BST film thickness.

$\left(T_{c}^{*}\right)$ were monitored as a function of film thickness (Fig. 6). The collapse in $T_{c}^{*}$ as film thickness decreased mirrored the collapse in $T_{m}$ (Fig. 3).

A tempting interpretation is (i) that the peak in dielectric constant $\left(\right.$ at $\left.T_{m}\right)$ is a Curie anomaly associated with the paraelectric-ferroelectric phase transition in the system; (ii) that the temperature at which the paraelectric-ferroelectric phase transition occurs is suppressed by reducing film thickness.

However, the care needed in the interpretation of CurieWeiss plots has been well discussed by Vendik et al. ${ }^{18}$ They point out the importance of the observation that the measured dielectric constant as a function of film thickness follows the so-called "series-capacitor" model:

$$
\frac{d}{\varepsilon_{\text {eff }}}=\frac{d}{\varepsilon_{\text {bulk }}}+K
$$

where $d$ is the thickness of the dielectric layer, $\varepsilon_{\text {eff }}$ is the measured dielectric constant, $\varepsilon_{\text {bulk }}$ is the inherent dielectric constant of the thin film material, and $1 / K$ is a constant parasitic "interfacial capacitance" that acts in series with the film, and whose origin is still a matter of considerable

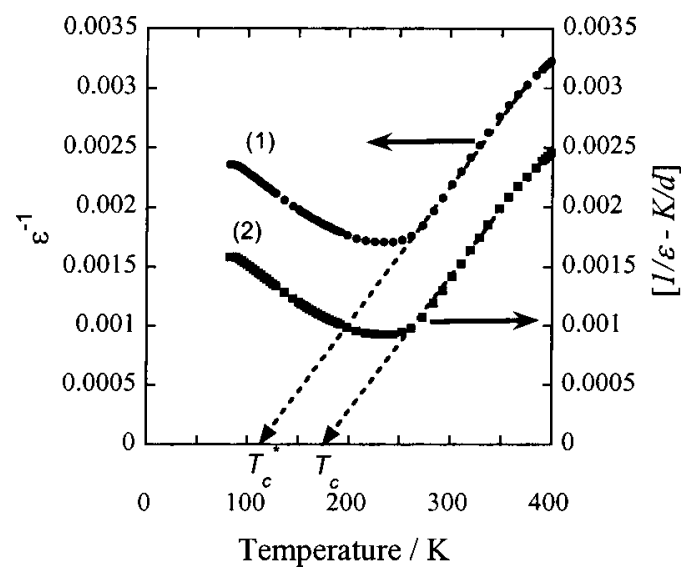

FIG. 5. Curie-Weiss plot taken directly from the measured temperature dependence of the dielectric constant (1), and also after a correction for the "interfacial capacitance" in the system (2).

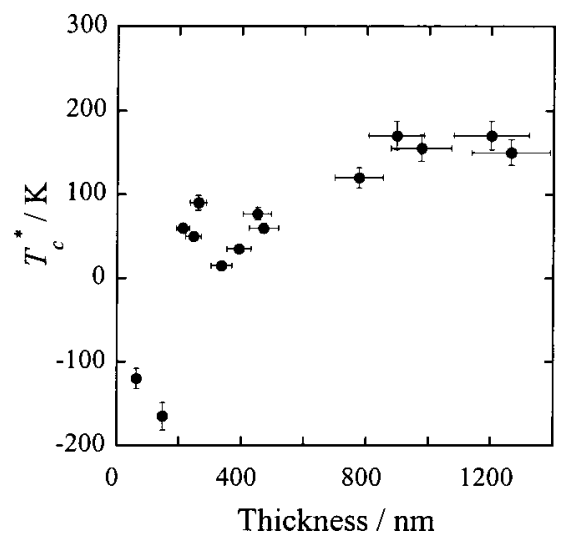

FIG. 6. Apparent Curie temperature as obtained from Curie-Weiss plots without taking interfacial capacitance into account.

debate. ${ }^{1,19-23}$ Whatsoever its origin, there is no doubt that the interfacial capacitance considerably affects the apparent $\mathrm{Cu}$ rie Temperature extracted directly from Curie-Weiss plots.

Reference to Eq. (3) indicates that determination of the inherent Curie Temperature, accounting for spurious interfacial capacitance effects, requires a plot of $\left[\left(1 / \varepsilon_{\text {eff }}\right)-(K / d)\right]$ against $T$ rather than $1 / \varepsilon_{\text {eff }}$ against $T$. To determine the interfacial capacitance, a "series capacitor plot" for this capacitor system was constructed (Fig. 7). Since the interfacial capacitance was temperature invariant (an observation taken from literature, ${ }^{6,11}$ but also by taking series capacitor plots across a range of temperatures for the films in this study), "corrected" Curie-Weiss plots were made, from which inherent Curie temperatures could be extracted. Figure 8 shows a comparison between the apparent and inherent Curie temperatures as a function of thickness. Clearly, the inherent Curie temperature does not suffer the same systematic collapse on reducing BST film thickness as is the case for the apparent Curie temperature. Rather it looks to be largely independent of film thickness, implying that once the interfacial capacitance has been accounted for, the paraelectricferroelectric phase change is not altered by thickness. It is worth noting that as the film thickness decreases, the func-

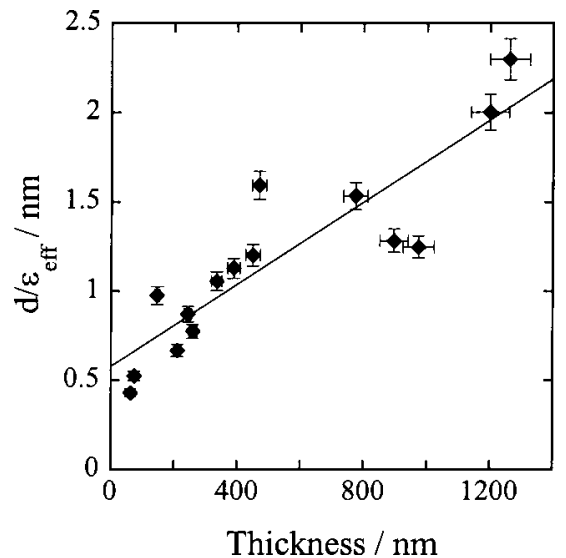

FIG. 7. $d / \varepsilon_{\text {eff }}$ as a function of $d$-the "series-capacitor plot," used to determine the interfacial capacitance in the system. This plot is for $10 \mathrm{kHz}$ at $150 \mathrm{~K}$, but a series of such plots at different temperatures showed the interfacial capacitance to be largely temperature and frequency independent. 


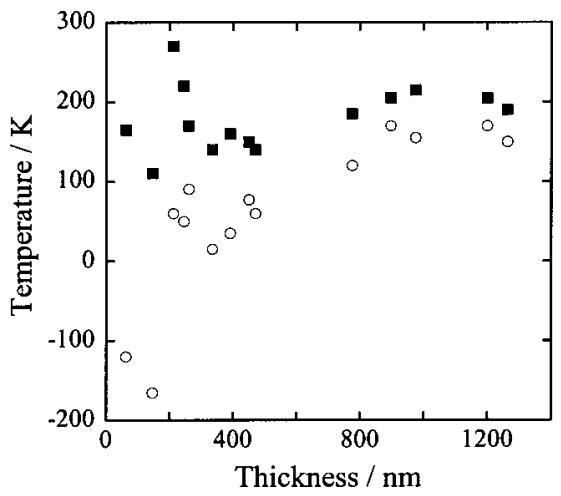

FIG. 8. Comparison between the apparent Curie temperature taken from Curie-Weiss plots of raw data (empty circles), and inherent Curie temperature after correction for interfacial capacitance (filled squares) had been performed. The corrected data set suggests that the inherent Curie temperature in the system is independent of BST film thickness.

tion $\left[\left(1 / \varepsilon_{\text {eff }}\right)-(K / d)\right]$ becomes strongly sensitive to the value taken for $K$, hence the greater scatter observed in Fig. 8 in the corrected $T_{c}$ as $d$ becomes small.

Polarization loops were taken at various temperatures between $80 \mathrm{~K}$ and $250 \mathrm{~K}$, and initially they were analyzed in terms of fitting to a Landau-Ginzburg-Devonshire (LGD) expansion in polarization-an approach used in previous work. ${ }^{15}$ Essentially it is assumed that the derivative with respect to polarization of a LGD free energy can be given as

$$
\frac{\partial(\Delta G)}{\partial P}=E=c_{1} P+c_{3} P^{3}+c_{5} P^{5}+\cdots
$$

While this appears a simplistic approach, it has been shown to be appropriate, even when the form of the LGD expression is complex, and accounts for local variations in strain and polarization. ${ }^{15}$ Sections of the $P-E$ loops representative of the derivative of the free energy (i.e., from $+P_{r}$ to large positive field, and from $-P_{r}$ to large negative field) were fitted to the polynomial given by Eq. (4), and, as in previous work $^{6,8,15}$ it was noted that $c_{3}$ was positive, implying second order behavior. An example of the fitting is demonstrated in Fig. 9. The behavior of $c_{1}$ was then plotted as a function of temperature and found to behave reasonably linearly. The point at which the best fit line crossed the temperature axis

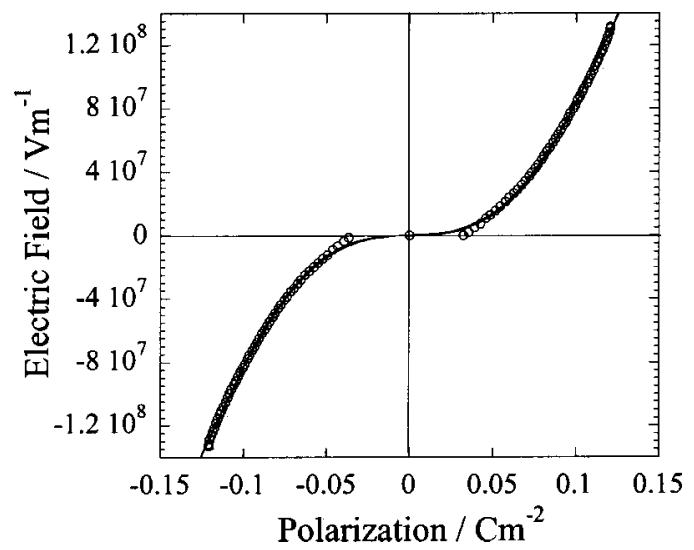

FIG. 9. An example of the LGD polynomial fit used to parametrize polarization loops.

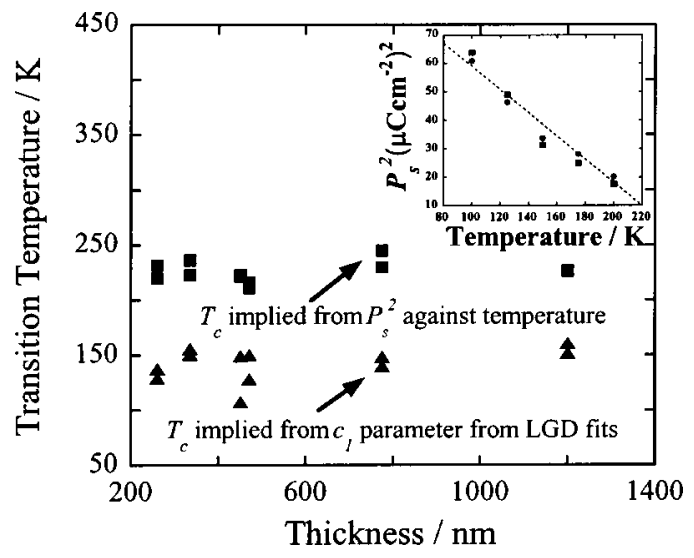

FIG. 10. The Curie temperatures implied by two different analyses of polarization loops with temperature, as a function of BST thickness, for films in which sound $P-E$ loops could be obtained. Inset: an example of the spontaneous polarization of one of the films as a function of temperature. Since the transition behavior was classed as second order, the intersection of the straight line of best fit with the temperature axis gave a representation of the Curie temperature.

(i.e., when $c_{1}$ changed sign) was taken as a representation of the ferroelectric-paraelectric transition temperature, and is shown in Fig. 10 as a function of thickness for all the films for which polarization loops could be obtained.

Further, since the transition was found to be second order, it was assumed that below $T_{c}$ the spontaneous polarization could be given as

$$
P_{s}^{2} \propto\left(T_{c}-T\right) .
$$

Thus $P_{s}^{2}$ (for both $+P_{s}$ and $-P_{s}$ ) was plotted against temperature for each capacitor, and the point at which the linear fit to the data crossed the temperature axis was taken as an alternative representation of $T_{c}$. This is also shown in Fig. 10 , and although the absolute value for $T_{c}$ is different from that taken from LGD loop fitting, it is clear in both cases that no thickness dependence of phase transition was implied from polarization loops.

Peaks in loss tangent are often observed associated with phase transitions in ferroelectrics, and in most of the films investigated here two loss peaks were observable (Fig. 11). The positions of the loss peaks, when clearly seen, were monitored as a function of film thickness (Fig. 11 inset). These also showed no thickness dependence.

Taking the evidence as a whole, it therefore seems that despite initial observations, there is no noticeable change in the ferroelectric-paraelectric phase transition temperature across the series of thicknesses of BST capacitors examined in this study. However, it must be noted that although consideration of the interfacial capacitance significantly affects the Curie temperature implied by Curie-Weiss analysis, it has absolutely no effect on the position of $T_{m}$ (see Fig. 5). We must therefore conclude that the position of $T_{m}$ is not simply related to phase transformation behavior in BST thin films. This observation means that the implications of previous experimental work, ${ }^{7}$ where the form of the dielectric constanttemperature response has been used to infer information about phase transition behavior in thin film systems, need to be considered with care. 


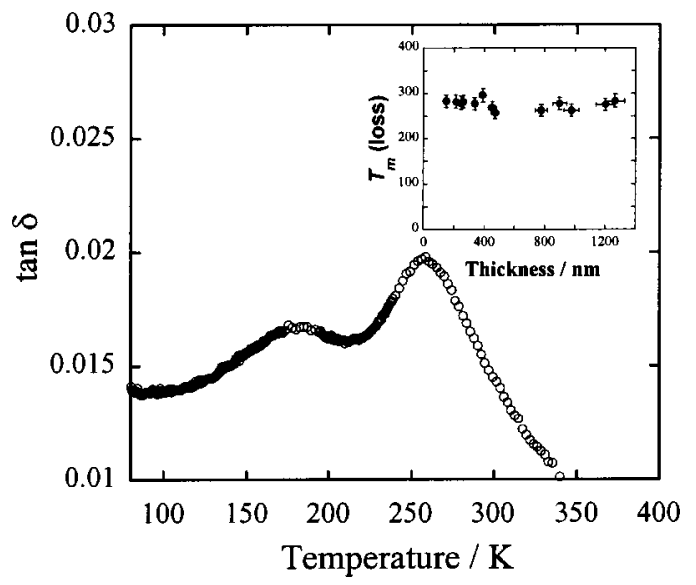

FIG. 11. Peaks in $\tan \delta$ with temperature were seen in almost all films. Inset: when the temperature of the larger peak was monitored, it showed no dependence on thickness (lower temperature peaks were less distinct).

So, how can the migration of $T_{m}$ to lower temperatures with decreased BST thickness be explained? Possible insight can be gained from consideration of the functional response of the BST capacitors when poled at $80 \mathrm{~K}$ prior to taking low-field dielectric constant data on heating (Fig. 12). Such low temperature poling changed the dielectric response from a single peak to a double or triple peak, as well as giving greater distinction to the peaks in the measured loss tangents. From these observations it is proposed that these anomalies are associated with phase transitions in the system. ${ }^{24,25}$ For this composition of BST (0.5-0.5), the bulk transition temperatures are expected as $\sim 235 \mathrm{~K}$ (cubic-tetragonal), $\sim 195$ $\mathrm{K}$ (tetragonal-orthorhombic), and $\sim 155 \mathrm{~K}$ (orthorhombic-rhombohedral), ${ }^{26}$ and these temperatures are approximately reflected in many of the anomalies in the poled thin films [see particularly Fig. 12(b)].

When the positions of the peaks in both dielectric constant and loss for poled films were monitored as a function of BST film thickness, they were found to be thickness invariant (Fig. 13) - consistent with the apparent thickness invariant nature of the Curie temperature discussed above.

In fact, even in the unpoled virgin state, for films below $150 \mathrm{~nm}$, a change in the temperature dependence of the dielectric constant from a single anomaly to two was observed (Fig. 14), mirroring the observations on poled samples. There is a significant difference between the Fermi energies of LSCO and Au electrodes, which produces a considerable internal bias in the system. Capacitance-voltage $(C-V)$ measurements on the LSCO/BST/Au system presented here indicated an internal bias of around $1.1 \mathrm{~V}$ (consistent with the Fermi level difference ${ }^{27}$ ). It is a possibility that the internal bias has become significant enough in the thinnest films to cause self-poling, and as a consequence, a change from a single broadened anomaly to two distinguishable anomalies.

In any case, across the entire series of observations, when more than one peak in the dielectric constant as a function of temperature was observable (either through low temperature poling or self-poling) the relative magnitude of the dielectric constant associated with the highest and lowest temperature anomalies was found to change significantly as
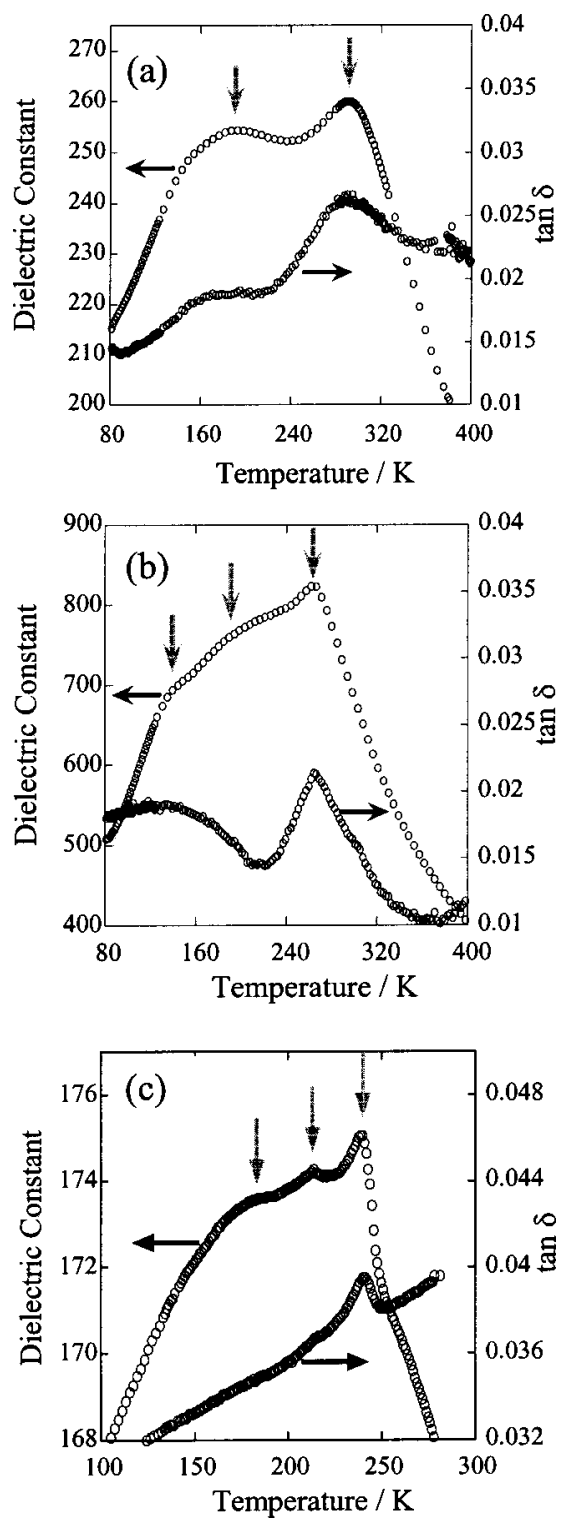

FIG. 12. Dielectric constant and loss tangent as a function of temperature for two PLD films (a) $260 \mathrm{~nm}$ and (b) $975 \mathrm{~nm}$ after poling at $80 \mathrm{~K}$ prior to data collection on heating. Functional testing without poling revealed only one peak in dielectric constant, whereas poling revealed two clear peaks in thinner films, and the suggestion of three peaks in thicker films. In (c) three dielectric anomalies are obvious in the $175 \mathrm{~nm}$ CSD BST film that had been cooled to $30 \mathrm{~K}$ prior to collecting dielectric constant data on heating.

film thickness was reduced, such that the low temperature anomaly became increasingly important. As an illustration, for the data taken from poling due to an externally applied field, the low:high temperature anomaly height ratio is plotted as a function of thickness in Fig. 15. The increasing strength of the low temperature anomaly contribution to the dielectric constant-temperature response on reduced film thickness is entirely consistent with the temperature shift in $T_{m}$ observed in virgin films. The reason for the relative change in strength of anomaly contributions demands further research.

\section{Summary of observations on PLD films}

The functional properties of thin film barium strontium titanate capacitors made by PLD revealed the following as 

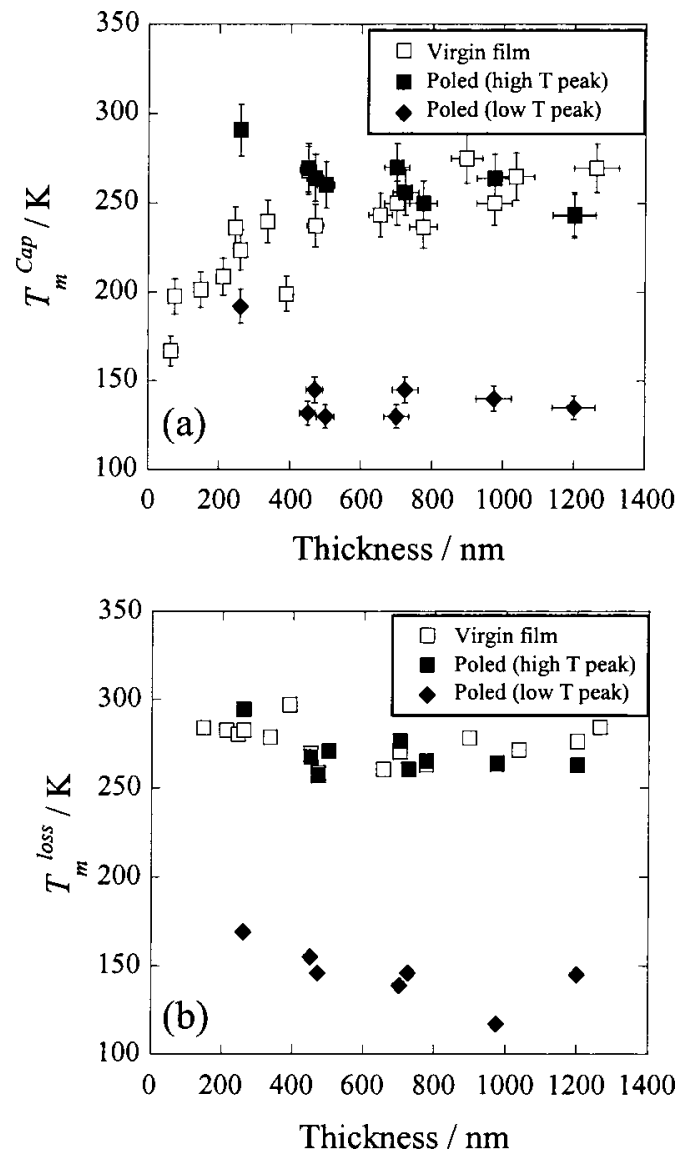

FIG. 13. The temperature at which peaks in dielectric constant (a) and loss tangent (b) were observed for the films in their virgin state, and after poling at $80 \mathrm{~K}$. In the poled data in (a) and (b) only the lowest and highest temperature peaks observable for each film are plotted.

film thickness was reduced: (i) there was a collapse in the dielectric constant; (ii) the "Curie anomaly" broadened and $T_{m}$ migrated to lower temperatures; (iii) the Curie temperature taken directly from measured data decreased.

It was tempting to suggest that the paraelectricferroelectric phase transition in the system was being depressed as a result of decreasing thickness, however, polarization measurements, peaks in loss tangents, and the correction of the Curie-Weiss analysis for interfacial capaci-

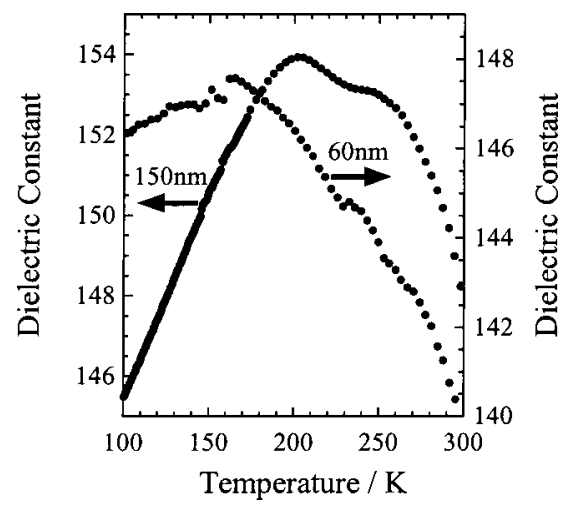

FIG. 14. Dielectric constant as a function of temperature for the $150 \mathrm{~nm}$ and $60 \mathrm{~nm}$ BST films (at $10 \mathrm{kHz}$ ). For these thinner films, rather than display a single anomaly, two anomalies were observable.

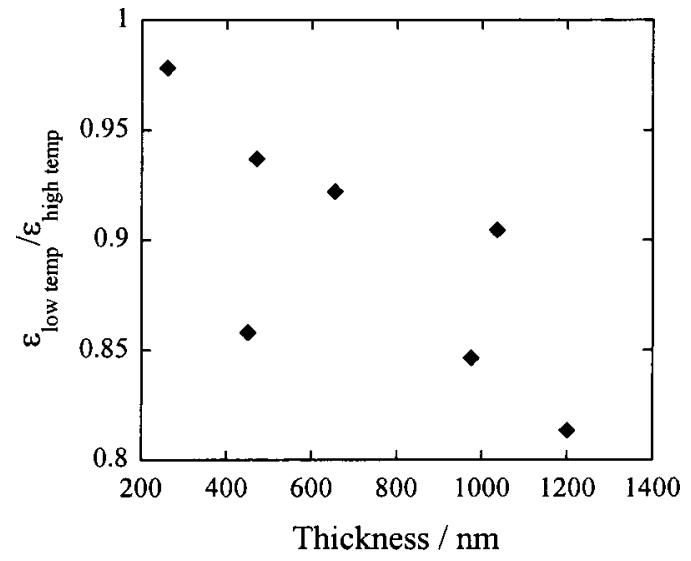

FIG. 15. Ratio of the height of the lowest temperature peak observed in dielectric constant to the highest temperature peak observed after poling. Clearly the relative strengths of these peaks changes reasonably systematically with thickness.

tance suggested that this was not the case, and that in fact phase transition behavior was thickness invariant. Further, since the migration of $T_{m}$ was not affected by corrections for interfacial capacitance, it can be concluded that $T_{m}$ is not simply related to phase transformation behavior in BST thin films. Instead, from examination of the dielectric response of poled films, the migration of $T_{m}$ was thought to be related to a change in the relative strengths of low and high temperature anomalies as film thickness changed.

\section{B. Observations on chemical solution deposited films}

The kinds of trends revealed by examining the thickness dependence of functional response in PLD films outlined above could not be repeated in the CSD capacitors, since only films with a BST layer $175 \mathrm{~nm}$ in thickness were available for characterization. Nevertheless, the measured properties of these films served to confirm that the phase transition behavior may not be dramatically different from bulk [suggested above by the poled dielectric response for BST 0.50.5 shown in Fig. 12 (b)].

Figure 12(c) (Ref. 28) illustrates the behavior of the dielectric constant and loss tangent on heating from low temperature for the CSD films. Again three anomalies were observed, in this case at $\sim 185 \mathrm{~K}, \sim 215 \mathrm{~K}$, and $\sim 240 \mathrm{~K}$ (see Table I). For this composition (0.7-0.3), the bulk transition temperatures are expected at $\sim 180 \mathrm{~K}, \sim 235 \mathrm{~K}$, and $\sim 290$

TABLE I. Compilation of the temperatures of anomalies in dielectric constant observed in this study for $175 \mathrm{~nm}$ CSD films on cooling and heating, compared with data from Rarker et al. (Ref. 7) along with bulk single crystal data for $\mathrm{Ba}_{0.7} \mathrm{Sr}_{0.3} \mathrm{TiO}_{3}$.

\begin{tabular}{lcccc}
\hline \hline & Thickness/nm & \multicolumn{3}{l}{ Temperature of anomaly/K } \\
\hline Bulk 24,29,30 & $\sim \infty$ & 290 & 235 & 180 \\
On cooling: & $580^{7}$ & 269 & $\ldots$ & $\ldots$ \\
& $300^{7}$ & 253 & $\ldots$ & $\ldots$ \\
& 175 & 186 & $\ldots$ & $\ldots$ \\
& $68^{7}$ & 144 & $\ldots$ & $\ldots$ \\
On heating from $\sim 30 \mathrm{~K}$ & $40^{7}$ & 102 & $\ldots$ & $\ldots$ \\
\hline \hline
\end{tabular}




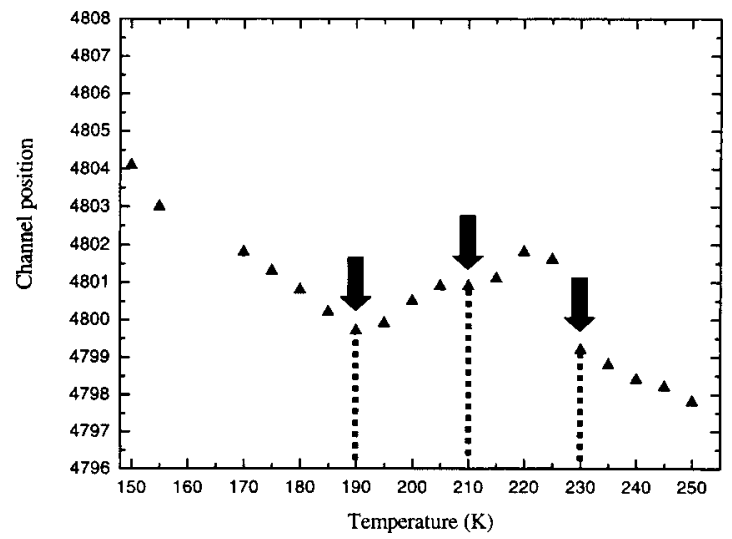

FIG. 16. Channel position for the 311 reflection from the CSD $175 \mathrm{~nm}$ BST film on heating. The presence of small but discernible anomalies in the lattice constant shows that the dielectric peaks in Fig. 12(c) cannot be artifacts from the electrical setup, nor caused by the electrode-BST interface physics.

$\mathrm{K} .^{24,29,30}$ Clearly, there are differences in the absolute temperatures of the apparent phase transitions, but the fact that three transitions are observed suggests that the resulting symmetry changes may well be the same as those in bulk. Temperature-variable x-ray diffraction studies (Fig. 16), on these films revealed distinct departure from linear thermal behavior at $\sim 190 \mathrm{~K}$, rejoining linear behavior at $\sim 230 \mathrm{~K}$. Between these temperatures, it is difficult to interpret additional phase change information, but a local strain minimum can be seen around $210 \mathrm{~K}$. Taking the evidence as a whole, it seems that the dielectric constant anomalies can be correlated with spontaneous strain features, and that therefore the dielectric peaks genuinely indicate phase transitions in this system.

Importantly, dielectric peaks were not always observed in these samples, and were only induced after cooling to cryogenic temperatures before heating. For samples that were only cooled to $175 \mathrm{~K}$, no sharp phase transition anomalies were evident on heating. This was also the case when the dielectric constant was monitored on cooling to $\sim 30 \mathrm{~K}$. In both cases, a broad peak with $T_{m} \sim 186 \mathrm{~K}$ was observed. The reasons for this behavior are not clear, and may indicate interesting kinetics in the system. However, it still seems likely that when the samples have been cooled to cryogenic temperatures, the subsequent phase transitions observed on heating are indicative of fundamental thermodynamics. Table I summarizes information on the values of $T_{m}$ found on cooling in the CSD films, combined with similar data taken from Parker et al. ${ }^{7}$ The data point for the $175 \mathrm{~nm}$ film studied here clearly fits the trend with decreasing thickness. Also in the table are temperatures of dielectric anomalies in bulk single crystal $\mathrm{Ba}_{0.7} \mathrm{Sr}_{0.3} \mathrm{TiO}_{3}$, along with the temperatures of anomalies observed in the $175 \mathrm{~nm}$ CSD film on heating from $\sim 30 \mathrm{~K}$.

\section{Implications for phase diagrams of BST thin films}

A definitive statement about the phase diagram of thin film BST implied is difficult to make from the data presented here. However, some discussion is warranted.
Relevant lattice parameters for consideration are LSCO: 3.81 $\AA$ (determined in this study), Pt: $3.92 \AA, \mathrm{SrRuO}_{3}: 3.93 \AA$, $\mathrm{Ba}_{0.5} \mathrm{Sr}_{0.5} \mathrm{TiO}_{3}: 3.957 \AA$ (determined in this study), and $\mathrm{Ba}_{0.7} \mathrm{Sr}_{0.3} \mathrm{TiO}_{3}: 3.97 \AA \AA^{31}$ All combinations of the above electrode and BST materials should cause in-plane compression of the BST, with the associated stabilization of the $c$ phase as defined in literature ${ }^{13,14}$ for $(001)$ in plane orientations. In the case of $\mathrm{SrRuO}_{3}$ lower electrodes this stabilization of $c$ phase has been observed in previous work. ${ }^{8}$ However, here little evidence for similar $c$-phase stabilization is evident on LSCO, despite the out-of-plane $001_{\mathrm{LSCO}} \| 001_{\mathrm{BST}}$ orientation relation. The semicoherent nature of the LSCOBST interface could mean that strain relaxation has taken place, but the local interfacial strain state should involve regions of full coherence separated by misfit dislocations-a situation that Pertsev et al. ${ }^{13}$ tried to take into account by using an "effective substrate lattice parameter." We note, however, that there is a significant difference between a film that is able to relax from interfacial constraints (where lattice parameter is a function of thickness) and one that is constrained to a modified lattice parameter (assumed to be constant with thickness).

In this semicoherent picture of the LSCO-BST interface we would expect either slight negative misfit strain or none at all. In the former case, the predicted transition sequence from high to low temperature is paraelectric (cubic) to $c$ phase to $r$ phase to $a c$ phase, implying three phase transitions-observed here in relatively thick films. For thinner films only two transitions were evident, which could be predicted $^{13}$ by zero misfit strain in the transition sequence from high to low temperature of paraelectric to $r$ phase to $a c$ phase. However, we would expect the thinner films to experience less effective strain relaxation than thicker films, and hence expect the change from three to two anomalies to occur the other way around.

The behavior of the CSD films on $\mathrm{Pt}(111)$ has not yet been modeled theoretically, but clearly stabilization of a rhombohedral phase might be expected. Here, the reasonably close correspondence between the temperatures of dielectric anomalies and phase transitions seen in bulk, suggest that no such alterations in the phase diagram have occurred. We note that the existence of the $a c$ phase predicted by Pertsev et al. is, however, not confirmed by more recent $a b$ initio calculations of Dieguez, Vanderbilt et al. ${ }^{32}$

\section{CONCLUSIONS}

In summary, the functional characterization of the two sets of barium strontium titanate thin film capacitors have revealed the following.

(i) When a single broad anomaly in the dielectric constant as a function of temperature is observed in virgin films, $T_{m}$ of this anomaly may not be strongly related to the temperatures at which phase transitions to thermodynamically stable states occur. Inference of the thermodynamics of ferroelectric thin film systems based on $T_{m}$ information alone is therefore not reliable.

(ii) When relatively sharp distinct peaks were observed in the dielectric constant as a function of temperature (in- 
duced by either low temperature poling or simply cooling to cryogenic temperatures), often three distinct features could be observed. This is consistent with the sequence of phase transitions in bulk, and may have implications for the validity of the phase diagrams for thin films developed and discussed in recent literature. ${ }^{13,14}$ Clearly, though, a definitive statement requires the symmetry of the thin film phases to be determined as a function of temperature.

\section{ACKNOWLEDGMENTS}

The authors acknowledge Dr. Susana Hoffman-Eifert for fabrication of the chemical solution deposited barium strontium titanate capacitors, and the Engineering and Physical Sciences Research Council for financial support.

${ }^{1}$ C. A. Mead, Phys. Rev. Lett. 6, 545 (1961).

${ }^{2}$ A. K. Tagantsev and I. A. Stolichnov, Appl. Phys. Lett. 74, 1326 (1999).

${ }^{3}$ N. A. Pertsev, J. Rodriguez Contreras, V. G. Kukhar, B. Hermanns, H. Kohlstedt, and R. Waser, Appl. Phys. Lett. 83, 3356 (2003).

${ }^{4}$ M. Dawber, P. Chandra, P. B. Littlewood, and J. F. Scott, J. Phys.: Condens. Matter 15, L393 (2003).

${ }^{5}$ A. V. Bune, V. M. Fridkin, S. Ducharme, L. M. Blinov, S. P. Palto, A. V. Sorokin, S. G. Yudin, and A. Zlatkin, Nature (London) 391, 874 (1998); S. Ducharme, V. M. Fridkin, A. V. Bune, S. P. Palto, L. M. Blinov, N. N. Petukhova, and S. G. Yudin, Phys. Rev. Lett. 84, 175 (2000).

${ }^{6}$ C. Basceri, S. K. Streiffer, A. I. Kingon, and R. Waser, J. Appl. Phys. 82, 2497 (1997).

${ }^{7}$ C. B. Parker, J. P. Maria, and A. I. Kingon, Appl. Phys. Lett. 81, 340 (2002).

${ }^{8}$ L. J. Sinnamon, R. M. Bowman, and J. M. Gregg, Appl. Phys. Lett. 81, 889 (2002)

${ }^{9}$ F. Tsai and J. M. Cowley, Appl. Phys. Lett. 65, 1906 (1994).

${ }^{10}$ J. Junquera and P. Ghosez, Nature (London) 422, 506 (2003).

${ }^{11}$ J. F. Scott, Ferroelectric Memories (Springer, Berlin, 2000).

${ }^{12}$ A. I. Kingon, J.-P. Maria, and S. K. Streiffer, Nature (London) 406, 1032 (2000).

${ }^{13}$ N. A. Pertsev, A. G. Zembilgotov, and A. K. Tagantsev, Phys. Rev. Lett. 80, 1988 (1998).
${ }^{14}$ Z.-G. Ban and S. P. Alpay, J. Appl. Phys. 91, 9288 (2002).

${ }^{15}$ L. J. Sinnamon, J. McAneney, R. M. Bowman, and J. M. Gregg, J. Appl. Phys. 93, 736 (2003).

${ }^{16}$ In the article by Parker et al. (Ref. 7), the authors fit their dielectric data as a function of temperature to an equation of the form: $(1 / \varepsilon)=\left(1 / \varepsilon_{\max }\right)$ $+\left[\left(T-T_{\max }\right) / 2 \varepsilon_{\max } \delta^{2}\right]$, from which they extract a "diffuseness" parameter $\delta$. The use of this equation is an unfortunate consequence of a typographical error in the article by Smolenski (Ref. 17) from which the equation is taken. It originates from the expression for inverse permittivity $(1 / \varepsilon)$ $=\left(1 / \varepsilon_{\max }\right) \exp \left[\left(T-T_{\max }\right)^{2} / \delta^{2}\right]$ which can be reexpressed using the series expansion for the exponential as

$\frac{1}{\varepsilon}=\frac{1}{\varepsilon_{\max }}\left(1+\frac{\left(T-T_{\max }\right)^{2}}{2 \delta^{2}}+\frac{\left(T-T_{\max }\right)^{4}}{8 \delta^{4}}+\cdots\right)$.

This is then approximated to

$\frac{1}{\varepsilon} \approx \frac{1}{\varepsilon_{\max }}\left(1+\frac{\left(T-T_{\max }\right)^{2}}{2 \delta^{2}}\right)$.

In Smolenski (Ref. 17), the squared power in the second term of the series expansion is omitted, but is presumably merely a typographical error as it is reintroduced later in the article.

${ }^{17}$ G. A. Smolenski, J. Phys. Soc. Jpn. S28, 26 (1970).

${ }^{18}$ O. G. Vendik and S. P. Zubko, J. Appl. Phys. 88, 5343 (2000).

${ }^{19}$ C. Zhou and D. M. Newns, J. Appl. Phys. 82, 3081 (1997).

${ }^{20}$ K. Natori, D. Otani, and N. Sano, Appl. Phys. Lett. 73, 632 (1998).

${ }^{21}$ J. F. Scott, Ferroelectrics 232, 25 (1999).

${ }^{22}$ A. A. Sirenko, C. Bernhard, A. Golnik, A. M. Clark, J. Hao, W. Si, and X. X. Xi, Nature (London) 404, 373 (2000).

${ }^{23}$ L. J. Sinnamon, M. M. Saad, R. M. Bowman, and J. M. Gregg, Appl. Phys. Lett. 81, 703 (2002).

${ }^{24}$ G. A. Smolenskii and K. I. Rozgachev, Zh. Tekh. Fiz. 24, 1751 (1954).

${ }^{25}$ L. A. Knauss, J. M. Pond, J. S. Horwitz, D. B. Chrisey, C. H. Mueller, and R. Treece, Appl. Phys. Lett. 69, 25 (1996).

${ }^{26}$ Landolt-Börnstein: Numerical Data and Functional Relationships in Science and Technology, edited by K. H. Hellwege, New Series Group III, Vol. 16(a) (Springer, Berlin, 1981).

${ }^{27}$ K. C. Park and J. H. Cho, Appl. Phys. Lett. 77, 435 (2000).

${ }^{28}$ J. Kut, BSc Honours thesis, Cambridge University, 2003 (unpublished).

${ }^{29}$ K. Bethe and F. Welz, Mater. Res. Bull. 6, 209 (1971).

${ }^{30}$ L. Benguigui, Phys. Status Solidi A 46, 337 (1978).

${ }^{31}$ M. McQuarrie, J. Am. Ceram. Soc. 38, 444 (1955).

${ }^{32} \mathrm{O}$. Dieguez and D. Vanderbilt (private communication). 